\title{
MANES SI SAPERENT... \\ REFERENTES REALES Y METAFÓRICOS \\ DEL TÉRMINO MANES \\ EN LA EPIGRAFÍA DE HISPANIA
}

\section{MANES SI SAPERENT... REAL AND METAPHORIC REFERENTS OF THE WORD MANES IN THE EPIGRAPHY OF HISPANIA}

\author{
Silvia Tantimonaco* \\ Universitat de Barcelona \\ silvia.tantimonaco@gmail.com \\ ORCID: 0000-0001-7060-0800
}

DOI: $10.1387 /$ veleia.18078

\begin{abstract}
Resumen: Ofrecemos en el presente artículo un análisis pormenorizado de las menciones de los Manes en las inscripciones de la Península Ibérica, excluyendo las dedicaciones Dis Manibus (sacrum), que son muy típicas de la epigrafía funeraria de todo el Imperio, y serán tratadas solo de forma muy general. Nuestro objetivo es doble: por un lado, definir mejor los referentes del término, profundizando así en el conocimiento de los Manes como seres del post mortem; por el otro, investigar sobre las modalidades y los tiempos de recepción y adopción de su culto en la provincia de Hispania.

Palabras clave: Léxico latino, Epigrafía latina, post mortem, Manes, CLE, religión romana.
\end{abstract}

Abstract: In this paper we offer a detailed study of the mentions of the Manes in the inscriptions of the Iberian Peninsula. The dedications Dis Manibus (sacrum), that are very common in the funerary epigraphy of the whole Roman Empire, will be treated only in general terms. The goal of our contribution is to provide a better understanding, on the one hand, of the meaning of the word Manes, creatures belonging to the Roman post mortem, and, on the other hand, of the process and chronology of reception and adoption of this cult in the province of Hispania.

Keywords: Latin Vocabulary, Latin Epigraphy, post mortem, Manes, CLE, Roman religion.

Recibido: 08-09-2016

Informado: 04-02-2017

Definitivo: 21-02-2017

* Universitat de Barcelona, becaria FI-DGR 2014 y miembro del grupo de investigación LITTERA (2014SGR63). Este trabajo se inserta en el proyecto de investigación FFI2015-68571-P. Quiero agradecer a los evaluadores anónimos las útiles correcciones y a la Prof. M. ${ }^{a}$ Cruz González Rodríguez, editora de la revista, la atenta revisión del texto en castellano. 


\section{INTRODUCCIÓN}

Los Manes, espíritus de los difuntos ${ }^{1}$, son conocidos en epigrafía sobre todo por la fórmula funeraria Dis Manibus, que aparece de manera casi serial en los epitafios romanos de la época imperial $^{2}$. En efecto, dicha fórmula empieza a documentarse en las inscripciones a partir de la edad de Augusto, encontrando una amplia extensión en el siglo ir y hasta la Antigüedad Tardía ${ }^{3}$. Va seguida normalmente por el nombre del difunto en caso genitivo o dativo, una construcción sintáctica que expresa posesión ${ }^{4}$ y permite emplear el sustantivo, que es plurale tantum, con el valor de un singular, referido al difunto individual en calidad de ser divinizado.

En este sentido, cabe pensar que el culto de los dioses Manes adquirió los caracteres del culto heroico de modelo helénico por imitación, a nivel popular, de las ceremonias establecidas por el propio Augusto en honor de algunos miembros de su familia, tal y como queda reflejado en la epigrafía oficial y en las fuentes historiográficas ${ }^{5}$. Es precisamente en la Eneida de Virgilio, máximo poeta de la edad augustea, donde Manes se emplea por primera vez con referencia a un individuo y en asociación con el culto de héroes como Anquises, Polidor o Deifobo; es ahí, además, donde los referentes semánticos de la palabra se extienden para abarcar nuevos significados trasladados, haciendo de Manes una palabra polisémica ${ }^{6}$.

En esta contribución, llevaremos a cabo un estudio léxico-semántico del término Manes, centrándonos en los materiales epigráficos de la Península Ibérica ${ }^{7}$ ocupándonos especialmente de la caracterización de este concepto cuando se usa fuera de la fórmula Dis Manibus ${ }^{8}$.

1 Sobre los Manes, véase Hild 1904 y Bömer 1936. En publicaciones anteriores hemos tratado algunas cuestiones relacionadas con la mención de estos seres tanto en literatura como en epigrafía, cf. Tantimonaco 2013, 2015 y 2016.

2 Para esta fórmula epigráfica, remitimos en particular al estudio de Herman 1998. Cf. también Tantimonaco 2013.

${ }^{3}$ Para los primeros testimonios de la fórmula $\mathrm{Dis} \mathrm{Ma}$ nibus en Roma, cf. Solin 1971, 35-36 y 36, n. 1 (también en Tantimonaco 2013, 264, n. 10 y 2016, 2, n. 3).

${ }^{4}$ Los dos casos son equivalentes en la construcción de esta fórmula para indicar posesión, cf. Herman 1998. Sobre el uso del dativo posesivo en los epígrafes, cf. también Id. 1965.

5 Para algunas de estas fuentes, cf. Tantimonaco 2016, 15-18.

${ }^{6}$ Cf. Tantimonaco 2016, 7-14.

7 Se echa todavía en falta la sistematización del léxico latino referido a lo sobrenatural y a los seres in- termedios (como lemures, larvae, lares, etc.), cf. Guzmán Almagro 2014. Cf. también Id. 2013, para un estudio específico sobre el término larva.

${ }^{8}$ Quedarán excluidas de nuestro corpus las siguientes inscripciones: CILA II/3, 927 (Tabula Siarensis), por ser un texto jurídico y oficial; CIL II/7 749, carmen cristiano, en el que la lectura de la palabra Manibus no es segura ni, a nuestro juicio, probable (sobre esta inscripción, véase Gil 1976, 562-565); y también CILA II/3, 893, pues la mención de los Manes en esta inscripción forma parte de una integración de laguna (1. 2: si modo si[nt Manes - - -]) que, a pesar de basarse en la comparación con otros epígrafes (como p.e. CLE 1323,1: Si modo sunt Manes) y con el original properciano (PROP. 4, 7, 1: Sunt aliquid Manes: letum non omnia finit) no garantiza su autenticidad. Por último, excluimos las inscripciones falsas y modernas: CIL II * $106=C I L$ $\mathrm{II}^{2} / 7 * 33 ;$ CIL II *107 = CIL II $2 / 7 * 34 ; C I L$ II *397 = CIL II $2 / 14,2 * 34$ y CIL II 3001 (sobre esta última, véase Carbonell Manils 2013). 


\section{LOS MATERIALES HISPANOS 9}

Fuera de la fórmula de consagración, los Manes se mencionan tan solo en seis epígrafes hispanos $^{10}$, en mayoría CLE, localizados entre la Bética (cuatro inscripciones) y la Citerior (dos inscripciones) mientras que en Lusitania, por el momento, no contamos con ningún testimonio. Son precisamente estos seis epígrafes que contienen el término Manes declinado en alguna de sus formas los que aquí nos interesan. Comenzaremos su comentario por los hallazgos de la Citerior y continuaremos con los de la Bética, ordenando las inscripciones siguiendo el criterio cronológico ${ }^{11}$.

\section{Citerior:}

C1. Iesso (Conventus Tarraconensis). Placa de caliza de Santa Tecla. 49,8 $\times 38 \times 3,9$. Ll. 4-11: dísticos elegíacos, con repetición de un pentámetro en el v.5. IRC II $76=I R C \mathrm{~V}, 065,68=C L E$ Hisp 115 = PELCatalans L1. Siglo II d.C. Fig. 1 .

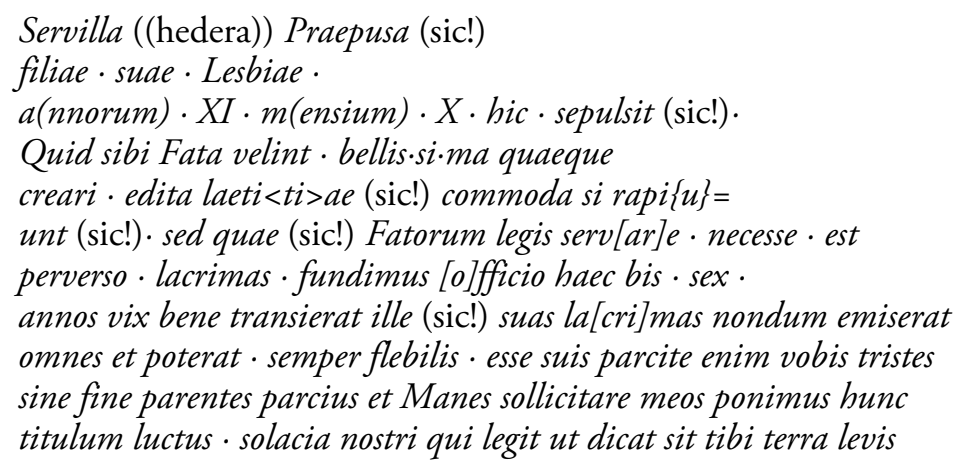

El sustantivo Manes, que aparece en la 1. 10 asociado a un adjetivo posesivo (Manes meos), designa en esta inscripción a la difunta en su estado post mortem. Las 11. 8-9, construidas a la manera de un discurso directo, podrían traducirse por: "Cuidad de vosotros, padres míos cuya tristeza no tiene fin, y dejad de molestar a mis Manes» ${ }^{12}$. Estamos ante un motivo tópico de los

9 Para todas las referencias bibliográficas y las abreviaturas de los corpora epigráficos, remitimos a la base de datos Clauss-Slaby (http://www.manfredclauss.de/ abkuerz.html).

10 Una búsqueda efectuada en la bases de datos Clauss-Slaby (n. 9) en fecha 12/06/2016 revela que, en Hispania, la fórmula Dis Manibus (sacrum) aparece en alrededor de 3365 inscripciones, repartiéndose en los porcentajes siguientes: $44,5 \%$ en la Citerior, $26,5 \%$ en Lusitania y $29 \%$ en la Bética (para la documentación en esta provincia, cf. Pastor Muñoz 2004, 387-388). Respecto a su cronología, estudios anteriores han evidenciado que, en la Península Ibérica, la dedicación a los Manes se registra ya a partir de la segunda mitad del siglo i d.C. en la Bética, difundiéndose especialmente a partir del siglo II d.C., cf. Lambrino 1965, 234; Pastor Muñoz 2004, 385; Id. 2006, 1428.
11 De cada inscripción, se señalarán los siguientes datos: localización geográfica; soporte; medidas; indicación de si es métrica o en prosa; referencia bibliográfica; cronología y, eventualmente, referencia fotográfica. Siguen la transcripción diacrítica del texto y un breve comentario, centrado sobre todo en la palabra Manes.

12 Cf. la traducción ofrecida en IRC (p. 115): "Ménagez vous, mes parents dont la tristesse est sans limite, et cessez d'implorer mes Mânes». Sin embargo, Gómez Pallarès (en PELCatalans, p. 48) considera también la posibilidad de que sollicitare sea un infinitivo pro imperativo y que, por lo tanto, parcite rija un infinitivo subtendido (esse, referido a tristes) de manera que sollicitare sería una exhortación y no una prohibición. 


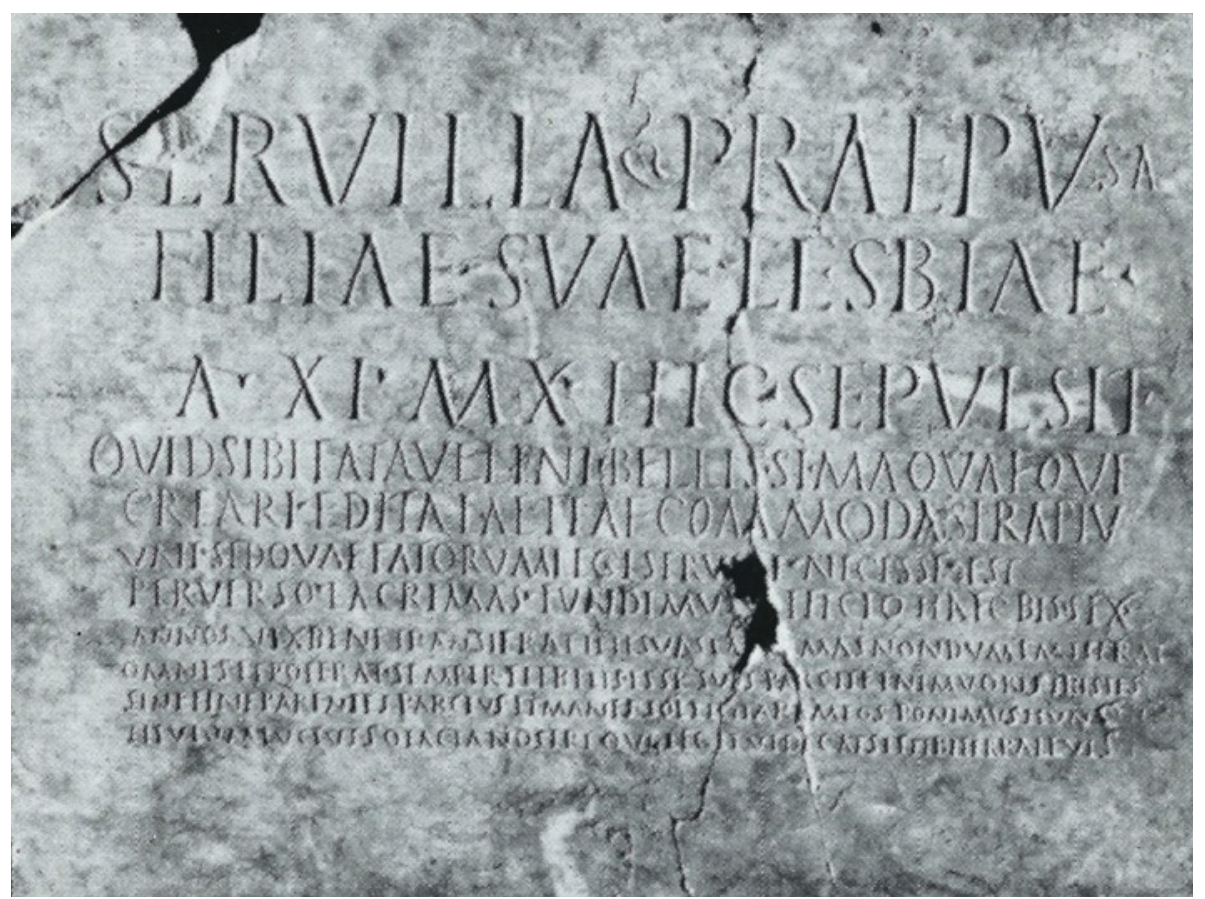

FiguRA I (fuente: IRC V)

CLE, en base al cual el difunto expresa molestia y turbamiento a causa de los lamentos de los vivos $^{13}$.

La producción de este monumento se inserta en un contexto libertino cultivado: desde el punto de vista textual, abundan las faltas ortográficas y las incongruencias; sin embargo, en lo que concierne al contenido, es evidente el influjo de los poetas, especialmente elegíacos ${ }^{14}$. De hecho, la propia oración parcite enim vobis tristes / sine fine parentes parcius et Manes sollicitare meos está inspirada en OV. Trist. 3, 11, 32: Parce, precor, Manes sollicitare meos ${ }^{15}$, al igual que en otras inscripciones en verso, como CLE 468, 2 (Cirta, Numidia): Parce pios Manes sollicitare manu, CLE 1198, 11-12 (Lugdunum, Gallia Lugdunensis): Manes / parcite iam luctu sollicitare meos; y CLE 965, 7-8 (Roma): Quid lacrumis opus est, Rusticelli carissime coniunx, / exstinctos cineres sollicitare meos? ${ }^{\text {?6. }}$.

13 Cf. Hernández Pérez 2001, 84-85.

${ }^{14}$ En general sobre la influencia de los poetas elegíacos en las inscripciones, cf. Lissberger 1934; para las citas virgilianas, cf., sin embargo, Hoogma 1959.

15 Cf. Thigpen 1995, 138; Hernández Pérez 2001, 85. Cf. también TIB. 1, 1, 67-68: Tu Manes ne laede meos; sed parce solutis / crinibus et teneris, Delia, parce genis y PROP. 2, 13, 32: Accipiat Manes parvula testa meos.

${ }_{16}$ También la locución sine fine, que aparece en la misma oración, es de uso exclusivamente poético y ca- racterística de Ovidio, quien, de hecho, la suele emplear en el segundo hemistiquio del hexámetro y con referencia al dolor (cf. OV. Pont. 1, 10, 23: Mei sine fine dolores; Am. 2, 10, 11: Meos sine fine dolores, etc.). Se repite en numerosos epitafios métricos, de entre los cuales el más parecido al nuestro sería un epígrafe de Roma casi contemporáneo (siglos i/II d.C.), CLE 1173, 1: Flet domus et cari lugunt sine fine parentes. Cf. Hernández Pérez 2001, 77-78. 
C2. Tarraco (Conventus Tarraconensis). Perdida, soporte desconocido. L1. 3, 5 y 7: hexámetros dactílicos irregulares. CIL II $4427=C I L \mathrm{II}^{2} / 14,21089=C L E 542=$ RIT 228. Siglo II-III d.C.

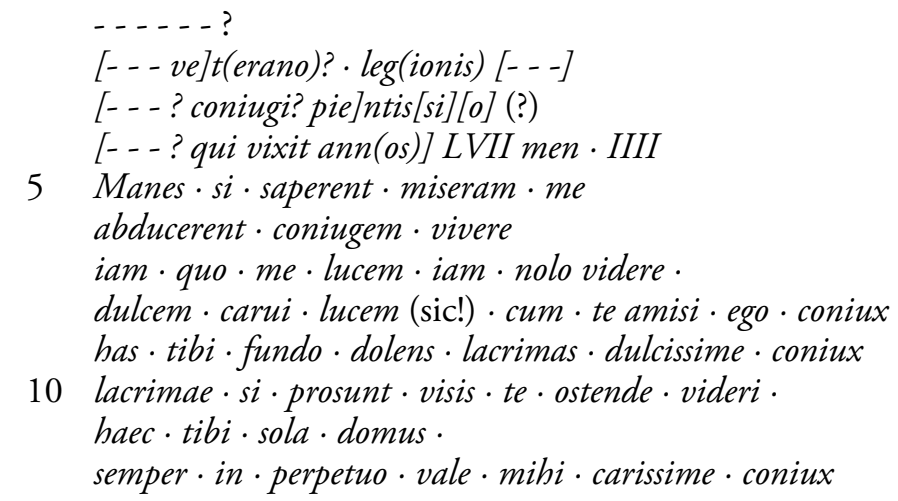

En este caso, nos encontramos ante la inscripción de un veterano, en la que la alusión a los Manes, ubicada en la 1.5, se inserta en el topos del taedium vitae, por el que los dedicantes desean dejar de vivir tras la muerte de sus seres queridos ${ }^{17}$. Los Manes, de hecho, son representados como los agentes de la muerte, quienes arrebatan injustamente a las personas de los suyos y del mundo: "Si los Manes fuesen más sabios, me habrían llevado consigo también a mí, su pobre esposa» ${ }^{18}$. Los Manes se presentan, pues, en esta inscripción con un carácter genérico e impersonal. Corresponden, de facto, a los dioses Ínferos, como demuestra la comparación con otras inscripciones, en las que la misma expresión de dolor se presenta en formulaciones tales como $S i$ quid (quicquam) sapiunt Inferi, de origen yámbico, documentadas en los CLE a partir de la primera mitad del siglo I d.C. ${ }^{19}$ En este sentido, la variante referida a los Manes sería una adaptación al ritmo dactílico, lo que probaría, una vez más, la «intercambiabilidad», a nivel conceptual, entre Inferi y Manes $^{20}$.

Por otra parte, la identificación de los Manes con los dioses Ínferos tiene un apoyo literario importante, que hunde sus raíces en la época republicana ${ }^{21}$. De hecho, ya en un fragmento antiguo (Ateyo o Catón) citado por Festo (p. 57), los Manes están vinculados con el mundus, la fosa fundacional de la ciudad (Eius inferiorem partem veluti consacratam Dis Manibus) y, en Cicerón, se contraponen a los Superi y a los homines ${ }^{22}$. Además, al igual que en nuestra inscripción, en un pasaje del De rerum natura, los Manes, en calidad de Ínferos, cumplen la tarea de llevar las almas de los difuntos en el Hades, reflejando una creencia popularmente extendida ${ }^{23}$. El mismo concepto de Manes como Ínferos se encuentra también abundantemente representada en la poesía del princi-

17 Cf. Hernández Pérez 2001, 27-34.

18 Traducción de Fernández Martínez 1998, 293. Por otra parte, Hernández Pérez $(2001,29)$ considera que se trata de una oración interrogativa.

19 Cf. Hernández Pérez 2001, 29-30.

20 Para esta casuística formular, cf. Hernández Pérez 2001, 28-31.

21 Sobre este tema, cf. Tantimonaco 2016, 2-5.

22 Cf. Cic. top. 90, 7-10: Atque etiam aequitas tripertita dicitur esse: una ad Superos deos, altera ad Manes, ter- tia ad homines pertinere. Prima pietas, secunda sanctitas, tertia iustitia aut aequitas nominatur.

23 Cf. LUCR. nat. 6, 763-764: [...] animas Acheruntis in oras / deducere forte deos Manis inferne reamur. En otro pasaje, de hecho, Lucrecio habla de los Manes como destinatarios de los cultos funerarios y expresa desaprobación por estas creencias, cf. ibid. 3, 52-54: Et nigras mactant pecudes et Manibus divis / inferias mittunt multoque in rebus acerbis / acrius advertunt animos ad religionem. 
pado, a partir de Virgilio, quien emplea metafóricamente el término para designar también al lugar adónde las almas acuden tras la muerte y dónde los difuntos residen ${ }^{24}$.

Cabe señalar que nuestra inscripción es muy similar a otra documentada en la misma ciudad y de cronología parecida, en que se lee: Inferi si qua sapent (sic!), miserum me abducite patrem / iam carui lucem (sic!), qui te amisi, ego, nate $e^{25}$ y con el que se parece también por la cláusula de despedida Semper in perpetuo vale, mi k[ar]issime na[te], casi idéntica a la de nuestra inscripción (Semper in perpetuo vale mihi carissime coniux), que se puede comparar con CAT. 101, 10: Atque in perpetuum, frater, ave atque vale ${ }^{26}$. Tiene rasgos en común también con otro carmen hispano, procedente de Salaria, en el que aparece la formulación: Si qu[a] Inferi sapent (sic!), vi m[e] abduceres. Si me amasti, fac abducas ${ }^{27}$. Asimismo, contiene citas literarias que la vinculan con otros textos, como p.e. la oración lacrimae si prosunt, visis te ostendere videri, inspirada en OV. Ars 1, 659: Et lacrimae prosunt, lacrimis adamanta movebis, y repetida en CLE 995B, 21 (Roma): Nil prosunt lacrimae nec possunt fata moveri ${ }^{28}$. En nuestra inscripción, la cita se inserta en el motivo relacionado con el deseo de volver a ver al ser querido por lo menos en sueńo $(\text { visis })^{29}$.

\title{
Baetica:
}

B1. Corduba (Conventus Cordubensis). Ara ossuaria. $83 \times 30 \times$ ?? Texto en prosa. CIL II $2 / 7$ $397=$ CIL II 2255. 19 a.C. Fig. 2.

\author{
$C($ aio $) \cdot$ Sentio $\cdot$ Sat (urnino $) \cdot \operatorname{co}(n) s(u l e)$ \\ $K$ (alendis) $\cdot$ Sextilib(us) \\ Dei $\cdot$ Manes \\ receperunt \\ 5 Abulliam $\cdot N($ umeri $) \cdot l($ ibertam $)$ \\ Nigellam
}

La manera de aludir a los Manes en esta inscripción — donde son mencionados en la 1.3 - refleja la equivalencia establecida entre los conceptos de Manes e Inferi, de la que ya hemos tratado a propósito de la inscripción anterior (C2). De hecho, los Manes son presentados como los que acogen a la difunta en su nueva morada (1l. 3-6: «Los Dioses Manes acogieron a Abullia Nigella, liberta de Numerio»), estableciéndose así una contraposición implícita entre el mundo de los vivos y el de los difuntos. En este sentido, creemos que el uso del perfectum (receperunt), sumado a la indicación precisa de la fecha por medio de la datación consular, contribuye a evidenciar la sacralidad

\footnotetext{
24 Cf. Tantimonaco 2016, 7-8. Para el uso de Manes como sinónimo de Inferi tanto en literatura como en epigrafía, cf. Bömer 1936, 294-295 y 299.

25 Se trata de $C I L I^{2} / 14,31279$ (= RIT 441), para la cual remitimos también a la edición y detenido comentario de PELCatalans T3.

26 Cf. Hernández Pérez 2001, 169.

27 La inscripción es CILA III, 362, cuya lectura ha sido corregida por Stylow en HEp 4 (1998), 495. Sobre las similitudes entre las tres inscripciones hispanas, cf. Hernández Pérez 2001, 28-29, quien cree que las dos tarraconenses podrían ser obra del mismo autor.

28 Cf. Hernández Pérez 2001, 68.
}

29 En este sentido, es interesante observar que, en literatura, las manifestaciones paranormales de los difuntos suelen designarse por medio de términos como phantasma, idolon o effigies, mientras que Manes se reserva, al parecer, para indicar, especialmente en el contexto ritual, el espíritu de un difunto en concreto, a menudo en asociación con su nombre personal, pero no su manifestación física, cf. p.e. Guzmán Almagro 2014. En la Eneida, Manes no se emplea nunca para designar una aparición, pues para ello se prefieren otros sustantivos, como simulacrum (cf. p.e. VERG. Aen. 2, 772) o imago (cf. p.e. ibid. 5, 636, referido a un sueño). 


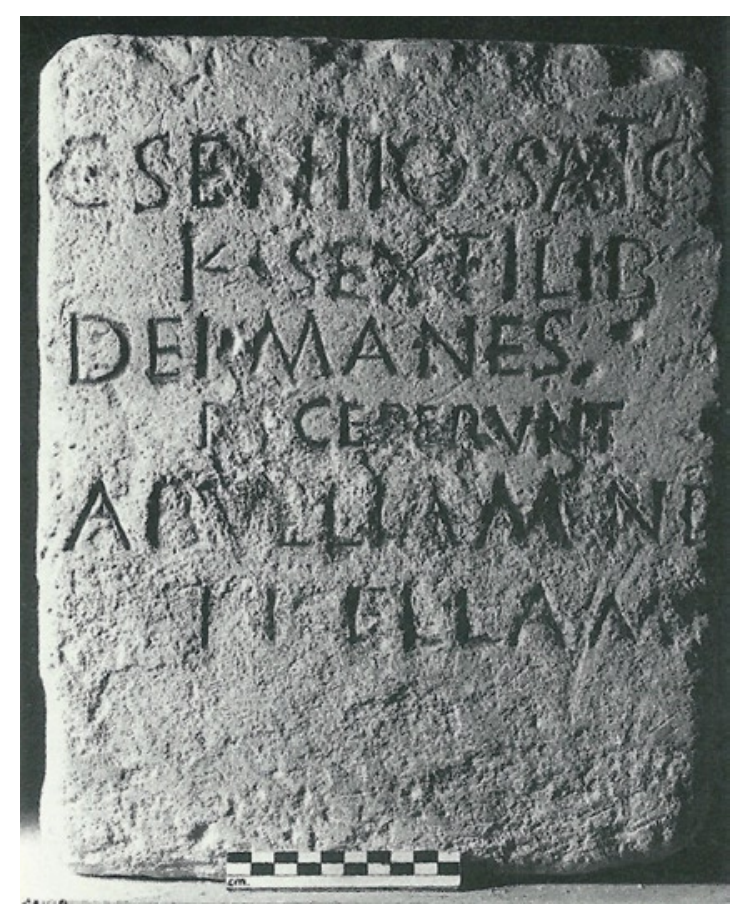

Figura 2 (fuente: CIL $I I^{2} / 7$ )

del acto de sepultura y la irrevocabilidad de la transformación de la difunta en Manes ${ }^{30}$. La inscripción, realizada en ámbito libertino, es, por ahora, la más antigua de Hispania y una de las más antiguas del mundo romano en que se mencionan los Manes $^{31}$.

B2. Seria (Conventus Hispalensis). Ara de mármol. $98 \times 31,5 / 26,5 \times 16$. Texto en prosa. ERBeturi 12. Mediados siglo I d.C. Fig. 3.

$D($ is $) \cdot M($ anibus $) \cdot$ s(acrum)
Cocc (eii) Mart $=$
ensis hom $($ o)
bon $($ us $)$ vix(it) ann(os)
$X X I I$ Ti(berius) Fl(avius) Felicio
pat $($ er $) \cdot$ fil(io) $\cdot$ pientis $($ simo $)$
fec(it) $\cdot$ si Manes
sapiunt $\cdot$ pater.
bene $\cdot$ LACHOS et
$Q V T \cdot A G A S N E($ sic!)

30 Cf. Tantimonaco 2015, 112-113.

31 De todos modos, este epígrafe llama la atención por su carácter totalmente excepcional tanto por lo que res- pecta al texto como a las letras y el soporte. Sobre ella, cf. Stylow 1995, 223-226; Id. 2002, 360-361. 


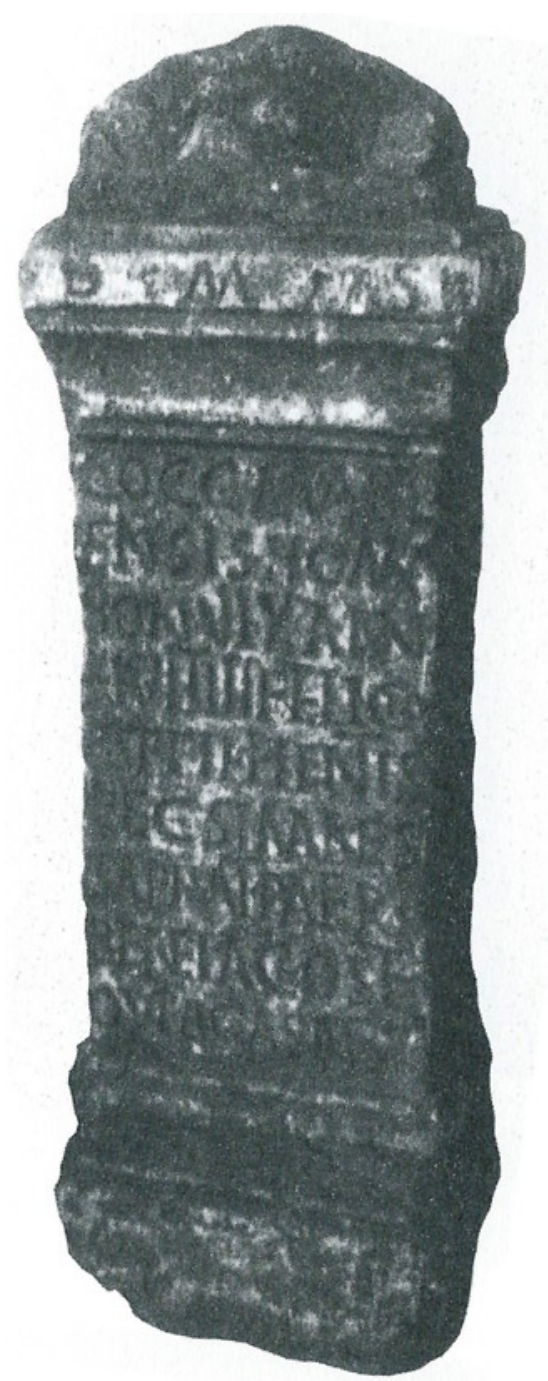

FIGURA 3 (fuente: ERBeturi 12)

Tal y como sucede en la inscripción C2, los Manes — que son mencionados en la 1.7, además que en la fórmula de consagración inicial - representan los agentes de la muerte y, al igual que en C2 y en B1, corresponden a los dei Inferi. De la misma manera que en la inscripción tarraconense, además, parece reprocharse a los Manes su manera de actuar injusta (11. 6-7: «Si los Manes fueran conscientes...»), pues se presenta aquí el mismo tipo formular Si quid sapiunt Inferi... No obstante, la imposibilidad de descifrar la parte conclusiva del texto no nos permite comentar más a fondo esta referencia ${ }^{32}$.

32 J. Gómez Pantoja, en HEp 7 (1997) propone la lectura siguiente: Si Manes / sapiunt, pater, / bene iace(ant) os(sa) et / quit agas, nes(ciant). 
B3. ¿Corduba? (Conventus Cordubensis). Placa de caliza. $(20) \times 36 \times 5$,5. El texto contiene un hexámetro con un pie excedente en la 1.1 y dos hexámetros seguidos, en lugar de hexámetro más pentámetro, en las 11. 5-6. CLEBetica C18 = CLEHisp 65. Finales del siglo I-principios del siglo II d.C. Fig. 4.

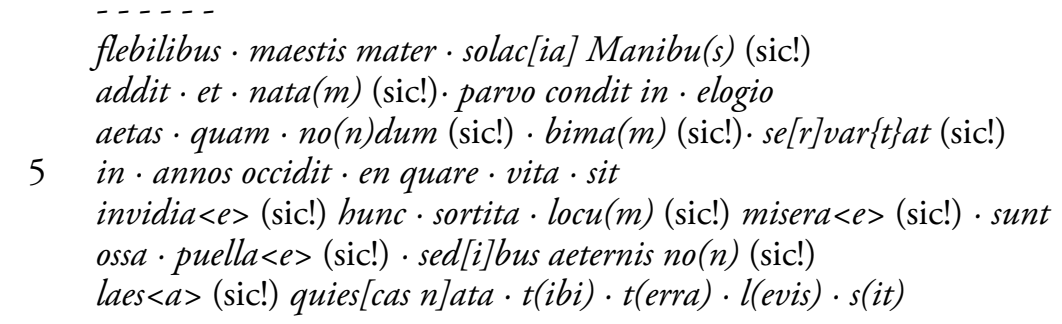

Frente a las inscripciones vistas hasta este momento, el término Manes (1.2) posee, en este texto del Conventus Cordubensis, un nuevo significado metafórico, pues se emplea directamente como sinónimo de «muerte», caracterizada como acontecimiento inconsolable (ll. 1-2: «A los Manes causa de llanto y tristeza la madre intenta dar consuelo y entierra a su hija en este pequeño monumento») $)^{33}$. De esta manera, la palabra adquiere el valor de un eufemismo que, en unión con los adjetivos flebiles y maesti, suscita la empatía y conmoción del lector. La representación del monumento funerario como elemento de consuelo ante la pérdida de un ser querido es un motivo tópico de las consolationes ${ }^{34}$. Por otra parte, la adjetivación maestis... Manibus encuentra paralelos literarios que encajan bien, por su cronología, con la posibilidad de haber influenciado la composición de nuestro texto (cf. SEN. Herc. f. 186s.: Maestos visere Manes y 648: Maestos ducat ad Manes vías) ${ }^{35}$ y que confirman el uso poético del término en nuestra inscripción.

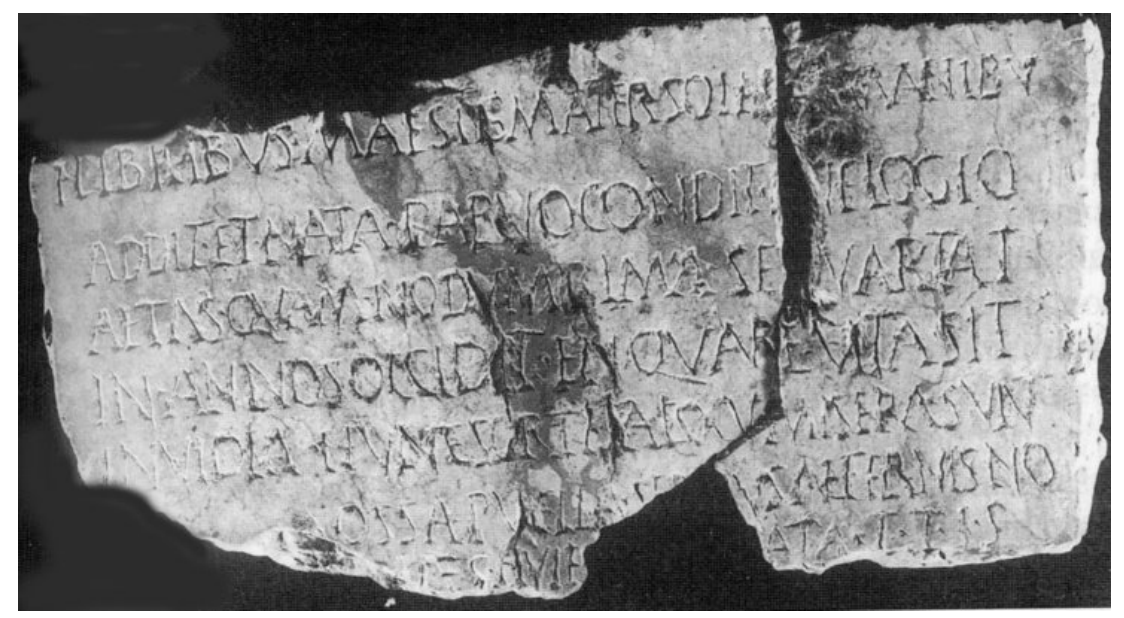

Figura 4 (fuente: CLEBetica C18)

33 Estas líneas, coordinadas por el et, representan un ejemplo de hysteron proteron, pues la secuencia lógica de las acciones es opuesta respecto al orden en que se expresan, cf. Hernández Pérez 2001, 124-125.
34 Cf. Hernández Pérez 2001, 121-130.

35 Cf. Fernández Martínez 2007, 209. 
B4. Corduba (Conventus Cordubensis). Estela de caliza. $(36,5) \times 23 \times 7-9$. Hexámetros dactílicos que empiezan en las ll. 5 (vos) y 7 (parcatis). CIL II $2 / 7569=$ CLEBetica C9 = CLEHisp 26. Finales del siglo iI d.C. Fig. 5.
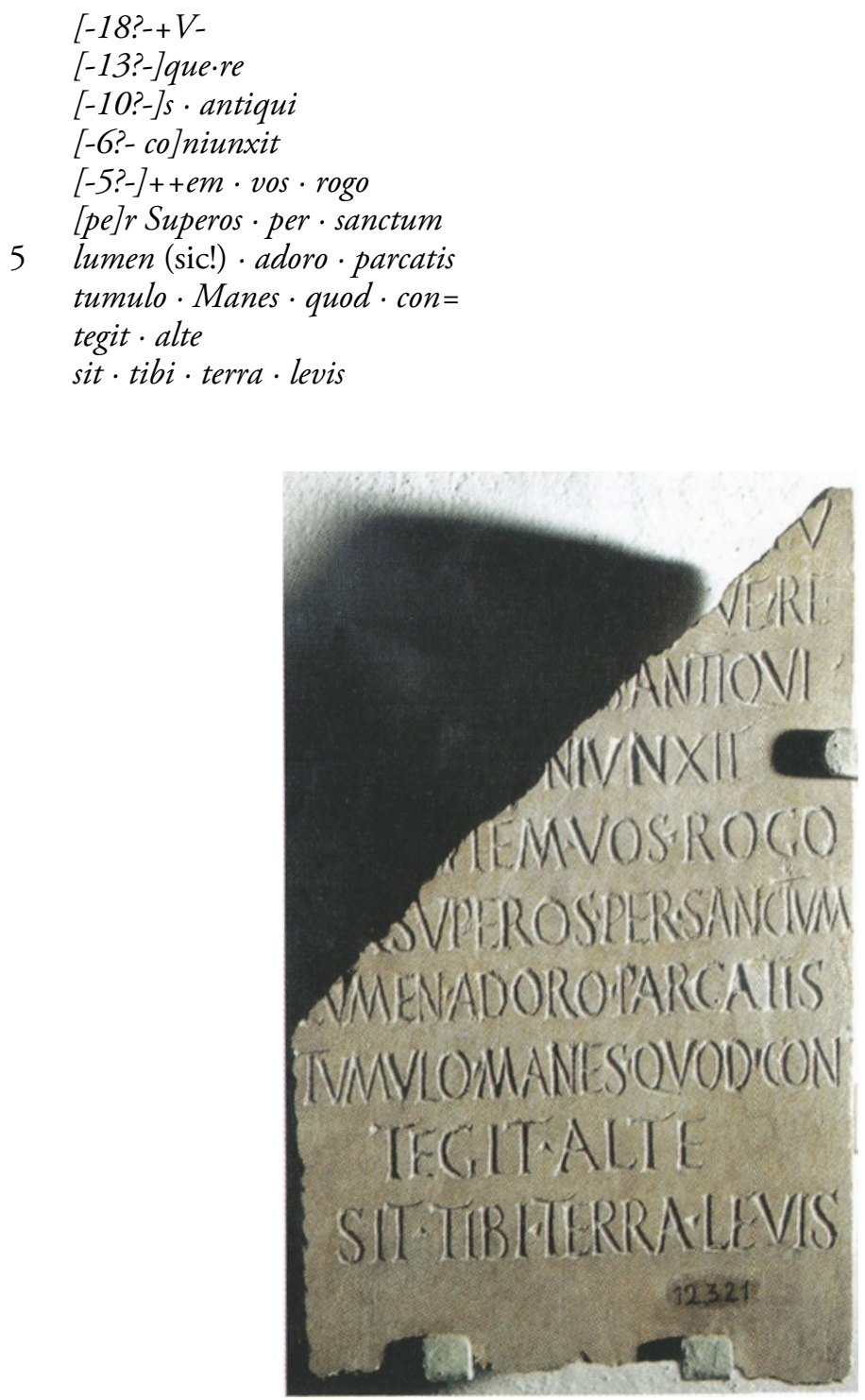

Figura 5 (fuente: CLEBetica C9)

La palabra Manes, que se encuentra en la 1. 6, se refiere, en este caso, a los restos mortales del difunto (11. 5-9: «Os imploro que respetéis la tumba que encierra en su seno estos Manes» ${ }^{36}$ ). Se trata

36 Traducción de C. Fernández Martínez 2007. 
de un significado que el término adquiere ya en dos pasajes de la Eneida, donde aparece en asociación con las cenizas (cf. VERG. Aen. 4, 34: Id cinerem aut Manis credis curare sepultos? e ibid. 427: nec patris Anchisae cineres Manisve revelli).

\section{Panorámica general}

Las inscripciones de Hispania que contienen mención a los Manes fuera del contexto formular conocido (Dis Manibus Sacrum) son principalmente carmina epigraphica. Es el caso de 2 sobre 2 textos procedentes de la Citerior y de 2 sobre 4 de la Bética. Los soportes, por otra parte, son especialmente placas realizadas con materiales de prestigio.

El análisis sociológico permite identificar con seguridad la presencia, entre los difuntos, de un veterano $(\mathrm{C} 2)$ y dos libertas $(\mathrm{C} 1$ y B1); en un caso (B2), nos encontramos ante un difunto y un dedicante con tria nomina, mientras que ignoramos el estatus de los restantes individuos (B3 y B4), a causa de las lagunas textuales.

En los textos analizados son frecuentes los elementos coloquiales y las grafías no normativas que reflejan el uso de la lengua oral —que hemos señalado en cada inscripción por (sic!). El dato más llamativo es que estas anomalías abundan en los epitafios de naturaleza literaria, cuya producción debió de enmarcarse en un contexto cultivado o, al menos, en el ámbito de una oficina epigráfica, siendo grabados, pues, por lapicidas profesionales (aunque cabe señalar que, también desde el punto de vista métrico, los textos no son impecables).

Para lo que concierne a la cronología, los epígrafes analizados abarcan desde el 19 a.C. (B1, epitafio no métrico) y mediados del siglo I d.C. hasta el siglo III, estando la mayoría de ellos fechados entre finales del iı y comienzos del III d.C. Desde el punto de vista geográfico, sin embargo, la distribución territorial evidencia que las escasas menciones de los Manes fuera de la fórmula Dis $\mathrm{Ma-}$ nibus se sitúan en las zonas de Hispania caracterizadas por una romanización más intensa y temprana, tal y como sucede en el caso de los conventus Tarraconensis y Cordubensis.

Fuera de la fórmula de consagración, el término Manes es empleado en las inscripciones hispanas para indicar múltiples conceptos y referentes. Así pues, designa especialmente a los Ínferos (C2, B1 y B2), en dos casos calificándolos como agentes de la muerte, como revela su mención dentro de la fórmula del tipo Manes si saperent (C2 y B2), que depende de una apódosis en la que se reprocha a los Manes el hecho de haber restado a los vivos sus seres queridos (en B2, dicha apódosis es sólo hipotética, a causa de la imposibilidad de descifrar el texto). Solo en un caso (C1) la palabra designa el espíritu de la difunta, entendido no como manifestación material (cf. supra, n. 29), sino como condición de la persona en el post mortem. Se trata del mismo referente que $M a-$ nes indica cuando en la fórmula Dis Manibus está asociado al nombre personal del conmemorado pero, en nuestra inscripción, el nombre personal es sustituido por el adjetivo posesivo: Manes... meos. En las inscripciones restantes, las acepciones son del todo metafóricas, pues, en un caso, la palabra Manes se utiliza como sinónimo de «muerte» (B3) y, en el otro caso, para indicar los restos mortales del difunto (B4).

\section{Conclusiones}

Todos los referentes que el término Manes designa en la epigrafía de Hispania se encuentran también en la literatura, especialmente en Virgilio y en los elegíacos de la edad augus- 
tea ${ }^{37}$. Es más, para las alusiones contenidas en los CLE de esta provincia es posible identificar una correspondencia exacta en las fuentes literarias (como hemos evidenciado para cada caso $)^{38}$. En este sentido, las más interesantes desde el punto de vista cultural son las inscripciones en prosa, pues testimonian de forma más espontánea la concepción de los Manes a nivel de creencias populares. Lamentablemente, contamos tan sólo con dos epitafios de este tipo, B1 y B4, respectivamente del año 19 a.C. y de finales del siglo II d.C. Sin embargo, este último, a pesar de estar en prosa, menciona los Manes en una expresión, Manes si sapiunt, que tiene como antecedente una fórmula yámbica de origen literario, mientras que en B1 los dei Manes corresponden a los dei Inferi, según una tradición consolidada.

En conclusión, la documentación hispana no presenta elementos originales en lo que concierne a la elaboración del concepto de Manes, pero sí hace gala, a pesar de los escuetos testimonios, de muchos de los múltiples referentes, reales y metafóricos, que el término había adquirido en el uso poético latino, y que debió de tener en el imaginario popular, desde la época de Augusto en adelante.

\section{BibLIOGRAFíA}

Bömer, F., 1936, s.v. Manes, en: Thesaurus Linguae Latinae, VIII.1, Lipsiae, 293-299.

Carbonell Manils, J., 2013, "CIL II 3001 (= CLE 1139), un epígrafe recention», en: C. Fernández Martínez et al. (eds.), Ex oficina. Literatura epigráfica en verso, Sevilla, 55-71.

Fernández Martínez, C., 1998, Poesía Epigráfica Latina, Madrid.

—, 2007, Carmina Latina Epigraphica de la Bética romana. Las primeras piedras de nuestra poesía, Sevilla.

GIL, J., 1976, «Epigraphica I», Cuadernos de Filología Clásica 11, 545-574.

Gómez Pallarès, J., 1992, "Poetas latinos como “escritores” de CLE», Cuadernos de Filología Clásica. Estudios latinos n. ${ }^{\circ}$ 2, 201-230.

Guzmán Almagro, A., 2013, «Demonios, máscaras y fantasmas: consideraciones sobre el término larva y sus significados», Emerita 81/1, 183-202.

—, 2014, «Latin Lexicon on ghosts», FIEC Conference 2014: http://fiec2014.sciencesconf.org/?lang=en.

Herman, J., 1998, «Dis Manibus. Un problème de syntaxe épigraphique», en: B. García Hernández (ed.), Estudios de lingüistica Latina. Actas del IX Coloquio Internacional de lingüistica latina (Universidad Autónoma de Madrid, 14-18 de abril de 1997), I, 397-408.

—, 1965, «Le datif possessif dans la latinité balkanique», en: P. Aebischer (ed.), Omagiu lui Alexandru Rosetti la 70 de ani, Bucureşti, 375-378.

Hernández Pérez, R., 2001, Poesía latina sepulcral de la Hispania romana: estudio de los tópicos y sus formulaciones, Valencia.

Hild, A., 1904, s.v. Manes, en: C.V. Daremberg, E. Saglio, Dictionnaire des Antiquités Grecques et romaines d'après les textes et les monuments, III, Paris, 1571-1576.

Hoogma, R.P., 1959, Der Einfluss Virgils auf die Carmina Latina Epigraphica. Eine Studie mit besonderer Berücksichtigung der metrisch-technischen Grundsätze der Entlehnung, Amsterdam.

Lambrino, S., 1965, "Les cultes indigènes en Espagne», en: A. Piganiol (ed.), Les Empereurs romains d'Espagne. Madrid - Italica, 31 Mars - 6 Avril 1964. Actes du Colloque Internationale sur les Empereurs romains d'Espagne, Paris, 223-242.

37 Cf. Tantimonaco 2016.

38 Con este respecto, cabe tener presente que también los poetas se inspiraban a veces en los epitafios para sus composiciones, cf. Gómez Pallarès 1992. 
Lissberger, E., 1934, Das Fortleben der Römischen Elegiker in den Carmina Epigraphica, Dissertation, Tübingen.

Pastor Muñoz, M., 2004, «Los dioses Manes en la epigrafía funeraria bética», Mainake 26, 381-394.

—, 2006, «Los Manes en la epigrafía de Mauritania Tingitana y Bética: diferencias y semejanzas», en: A. Akerraz et alii (a cura di), L'Africa romana. Mobilità delle persone e dei popoli, dinamiche migratorie, emigrazioni ed immigrazioni nelle province occidentali dell'Impero romano. Atti del XVI Convegno di Studio. Rabat, 15-19 Dicembre 2004, Roma, 1417-1438.

Solın, H., 1971, Beiträge zur Kenntnis der griechischen Personennamen in Rom, I, Helsinki-Helsingfors.

STYlow, A.U., 1995, «Los inicios de la epigrafía en la Bética. El ejemplo de la epigrafía funeraria», en: F. Beltrán (ed.), Roma y el nacimiento de la cultura epigráfica en Occidente. Actas del Coloquio "Roma y las primeras culturas epigráficas del Occidente Mediterráneo (siglos I a.E.-I d.E.)»(Zaragoza, 1992), Zaragoza, 219-238.

-, 2002, «La epigrafía funeraria en la Bética», en : D. Vaquerizo Gil (ed.), Espacios y usos funerarios en el Occidente romano. Actas del Congreso Internacional celebrado en la Facultad de Filosofía y Letras de la Universidad de Córdoba (5-9 de junio de 2001), I, Córdoba, 353-368.

Tantimonaco, S., 2013, «La formula Dis Manibus nelle iscrizioni della Regio X», en: F. Fontana (a cura di), Sacrum facere. Atti del I Seminario di Archeologia del Sacro. Trieste, 17-18 febbraio 2012, Trieste, 261278

—, 2015, “Singolari e plurali”, "maschili e femminili”. Alcune osservazioni sui Manes a partire dalle fonti epigrafiche», Erga-Logoi 3/1, 109-131.

—, 2016, «Stant Manibus arae. I Manes nell'Eneide di Virgilio», Anuari de Filologia. Antiqua et Mediaevalia 6, 1-21.

Thigpen, J. B., 1995, A literary analysis of Latin epitaphs from Roman Spain which contains creative biographical discourse, Ann Arbor. 